\title{
LOCALIZAÇÃO INDOOR BASEADA EM SISTEMAS RFID PARA O AMBIENTE INDUSTRIAL*
}

Matheus Jacon Pereira ${ }^{1}$ Leandro Avanço²

\section{Resumo}

Existe uma tendência de aumento na utilização da tecnologia de RFID na automação industrial, bem como em outros setores: varejo, aviação e transportes. Com o RFID, um objeto pode ser identificado por uma etiqueta eletrônica e sua existência constatada automaticamente. No entanto, sua localização exata permanece desconhecida para os usuários. Para a localização, os estudos realizados são satisfatórios quanto as técnicas de rastreamento de etiquetas RFID, porém, não consideram as possíveis interferências na comunicação provocadas pelos objetos presentes em locais reais. Este trabalho propõe um ambiente de laboratório que simula as interferências do Ambiente Industrial nas técnicas de localização indoor de etiquetas RFID, como obstáculos na comunicação entre etiquetas e antenas RFID; e materiais usados na fabricação das superfícies dos objetos, sobre as quais a etiqueta RFID é aplicada. Os resultados mostram que as técnicas RSSI e AoA são adequadas para localização indoor de etiquetas RFID, sendo que a segunda técnica permite maior exatidão na localização.

Palavras-chave: Identificação por rádio frequência; RFID; Localização indoor de etiquetas RFID; Automação; Indústria.

\section{INDOOR LOCATION BASED ON RFID SYSTEMS FOR THE INDUSTRIAL ENVIRONMENT}

\section{Abstract}

There is a tendency of an increase in the use of RFID technology in industry automation and management of storage of Products distribution centers, as well as in other sectors: retail, aviation and transport. With RFID, an object can be identified by an electronic tag and your existence established automatically. However, your exact location remains unknown to users. For the location, the studies are satisfactory as the techniques of tracking of RFID tags, however, do not consider the possible interference in communication caused by objects present in real locations. This work proposes a lab environment that simulates the interferences of the Industrial Environment in the techniques of indoor location of RFID tags, which are obstacles in communication between RFID antenna and tags; and materials used in the manufacture of the surfaces of the objects on which the RFID tag is applied. The results show that the techniques RSSI and AoA are suitable for indoor location of RFID tags, and the second technique allows greater accuracy in location.

Keywords: Radio Frequency Identification; RFID; indoor location RFID tags; Automation; industry.

1 Mestre em Engenharia da Computação, Centro de Tecnologia da Informação, Automação e Mobilidade do IPT - Instituto de Pesquisas Tecnológicas do Estado de São Paulo.

2 Mestre em Engenharia da Computação, Centro de Tecnologia da Informação, Automação e Mobilidade do IPT - Instituto de Pesquisas Tecnológicas do Estado de São Paulo. 


\section{INTRODUÇÃO}

$\mathrm{Na}$ automação industrial existe a necessidade de identificação rápida e a localização em tempo real de insumos, componentes e ferramentas, garantindo um monitoramento contínuo dos produtos [1].

Existe uma tendência de aumento na utilização da tecnologia de Identificação por Radiofrequência (Radio Frequency Identification - RFID) na automação industrial, bem como em outros setores: manufatura, varejo, aviação, indústria e transportes [2].

Com esta tecnologia, um objeto pode ser associado a uma etiqueta RFID exclusiva e sua existência pode ser detectada de forma automática. No entanto, sua localização exata ainda permanece desconhecida para o leitor de RFID e para os usuários que podem estar interessados no objeto [3].

Quando se trata da localização outdoor, o Sistema de Posicionamento Global (Global Positioning System - GPS) é frequentemente utilizado para localizar objetos. Ainda assim, esta tecnologia não é apropriada para rastreamento indoor, pois requer linha de visão direta entre o objeto e os satélites.

Para a localização indoor, existem técnicas de rastreamento de etiquetas RFID, que são classificadas de acordo com a característica do sinal de radiofrequência utilizada para o rastreamento. As duas técnicas mais consolidadas são: Variação da Intensidade do Sinal (Received Signal Strength Indication - RSSI) e Ângulo de Chegada (Angle of Arrival - AoA) [4].

Os trabalhos realizados por Brennan e Kolaja [5]; e; Zhou e Zhang [6] propõem métodos de localização baseados nas técnicas de rastreamento RSSI e AoA respectivamente. Os resultados destes trabalhos são satisfatórios, no entanto, não consideram as possíveis interferências na comunicação provocadas pelos objetos presentes em locais reais.

Como se sabe, a presença de objetos feitos de diversos materiais, como: metais, água, tecido, madeira e compostos plásticos, causam diferentes tipos de reflexão e absorção do sinal de radiofrequência, logo, ocasionam interferência na comunicação RFID [7].

O objetivo deste trabalho é realizar um estudo comparativo de técnicas de localização indoor de etiquetas RFID, avaliando os efeitos das interferências no ambiente industrial. As interferências são causadas por obstáculos na comunicação entre etiquetas e antenas RFID e por materiais usados na fabricação das superfícies dos objetos, sobre as quais a etiqueta RFID é aplicada. Este estudo é realizado por meio de ensaios experimentais em laboratório e pelo desenvolvimento de um Sistema de Localização de Etiquetas RFID.

Este trabalho está organizado de acordo com a breve descrição das seções a seguir. A Seção 2 apresenta os princípios básicos da tecnologia RFID. A Seção 3 discorre sobre os Sistemas de Localização em Tempo Real. A Seção 4 expõe como as interferências do ambiente influenciam o desempenho dos sistemas RFID. Já a Seção 5 propõe um Sistema de Localização de Etiquetas RFID, que estimará a posição de etiquetas RFID usando técnicas RSSI, AoA e ToA. A Seção 6 apresenta a proposta 
de ambiente laboratorial que simula as interferências do ambiente Industrial, os ensaios propostos e a análise dos resultados. Por fim, a Seção 7 mostra as conclusões.

\section{IDENTIFICAÇÃO POR RÁDIO FREQUÊNCIA}

O RFID pode ser definido como um sistema de identificação que utilizam radiofrequência para a comunicação, em que circuitos eletrônicos são anexados a objetos que se deseja identificar [8].

Entre os itens existentes em um sistema RFID, os dois mais importantes são: a etiqueta anexada ao objeto que se deseja identificar; e o leitor, dispositivo responsável por detectar a presença da etiqueta e interrogá-la para obter as informações armazenadas do objeto, usando uma ou mais antenas RFID (Figura 1).



Figura 1. llustração de um Sistema RFID.

As etiquetas são classificadas conforme sua fonte de energia. São denominadas ativas se possuírem uma fonte de alimentação e passivas na ausência deste recurso. Os sistemas podem operar em baixa frequência (LF) aproximadamente $125 \mathrm{kHz}$, alta frequência (HF) em torno de 13,56 MHz, ultra-alta frequência (UHF) em torno de 915 $\mathrm{MHz}$ e micro-ondas com 2,45 GHz e 5,8 GHz [9].

Este trabalho utiliza etiquetas passivas UHF, bastante populares pelo seu baixo custo, quando comparadas com etiquetas ativas e o desempenho desta frequência de operação (UHF) é melhor confrontado com faixas frequência mais baixas, principalmente com relação a distância máxima de leitura [5].

\section{SISTEMAS DE LOCALIZAÇÃO EM TEMPO REAL}

Do inglês Real-Time Locating System (RTLS), são sistemas automatizados que monitoram a atividade das etiquetas RFID presentes em seu campo de leitura. Desta maneira, permitem localizar os objetos em que estão anexadas, usando técnicas de rastreamento indoor. Dentre estas técnicas, as principais são a RSSI e a AoA.

\subsection{Variação da Intensidade de Sinal (RSSI)}

A resposta de uma etiqueta a uma interrogação é um sinal de radiofrequência com uma determinada intensidade (RSSI). Nesta técnica, o RTLS utiliza um mapa de intensidade de resposta previamente armazenado em sua memória para comparar o RSSI advindo da leitura das etiquetas, desta comparação, é realizado o cálculo das localizações [10]. 
Para os sistemas RFID passivos, em que o sinal de transmissão é retrorefletido (backscattered) pela etiqueta, a intensidade do sinal de resposta (RSSI) é determinada baseando-se nos ganhos da antena RFID, da etiqueta e do leitor, associados a eficiência de retroreflexão e distância entre antena do leitor e etiqueta [11].

\section{2 Ângulo de Chegada (AoA)}

Zhou e Zhang [6] apresentaram um método AoA de localização de etiquetas RFID UHF passivas, que utiliza a diferença de fase entre o sinal retrorefletido da etiqueta por duas antenas (Figura 2). Da diferença calculada, obtém-se um coeficiente e uma linha de direção. Utilizando-se dois pares de antenas podem-se obter duas linhas de direção. Da intersecção destas linhas, realiza-se a localização da etiqueta.



Figura 2. Arranjo experimental proposto por Zhou e Zhang [6].

\section{INTERFERÊNCIA DO AMBIENTE NOS SISTEMAS RFID}

Para o desenvolvimento de um sistema RFID eficiente, deve-se levar em conta a interação entre o sinal de radiofrequência e os materiais presentes nos locais da aplicação, pois obstáculos na comunicação entre antena e etiqueta podem causar interferências [7].

\subsection{Obstáculos na Comunicação}

Para a verificação desta influência, Bleda et al. [7] realizou um experimento que compara a eficiência de leitura com relação a obstáculos de materiais plásticos, metais e madeira comumente usados em mobiliário (Figura 3). A comparação mostrou os materiais que afetam mais as leituras de sistemas RFID. 


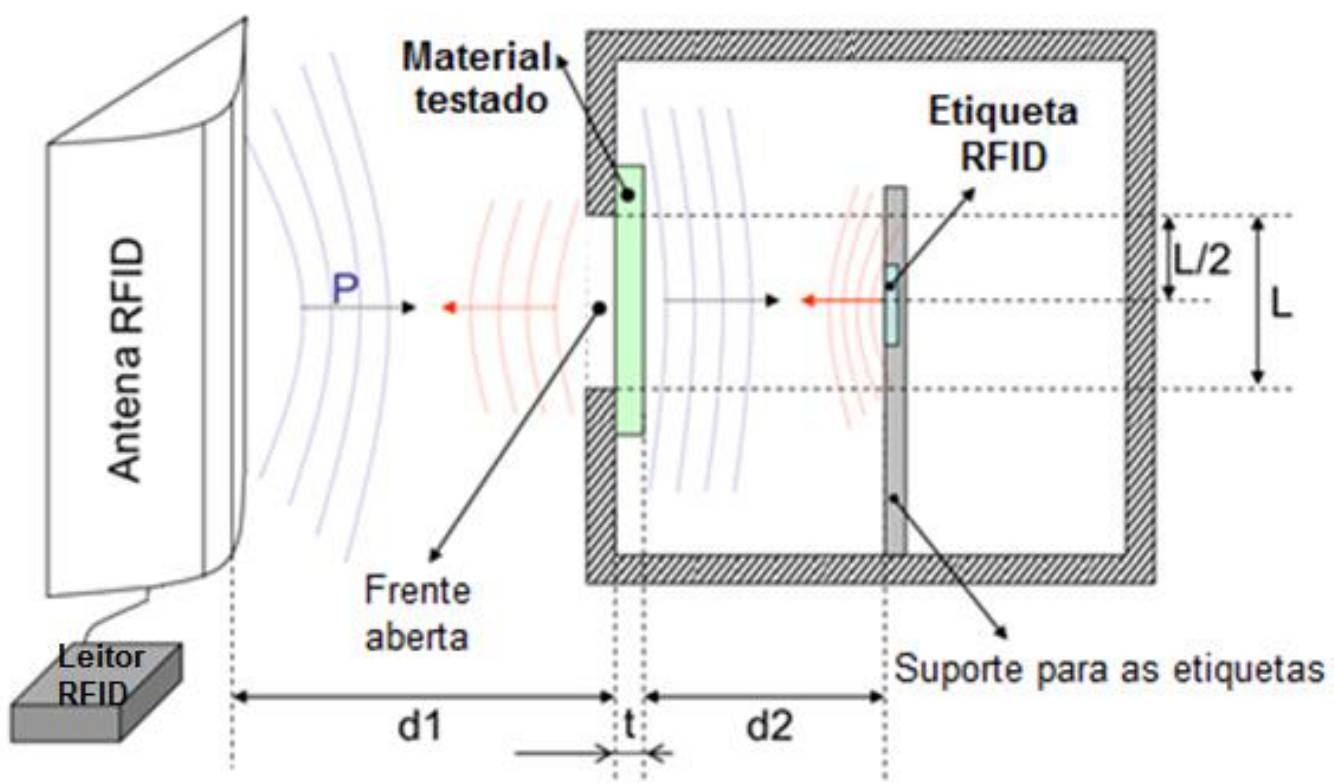

Figura 3. Arranjo experimental proposto por Bleda et al. [7].

\subsection{Superfície de Aplicação das Etiquetas}

Os obstáculos entre antena e etiqueta não são as únicas fontes de interferência possíveis. As etiquetas RFID precisam ser aplicadas na superfície do objeto que se deseja identificar e monitorar, as quais podem ser feitas de diversos materiais com diferentes características de constante dielétrica [12].

Mei e Yue [12] verificaram a influência da superfície de aplicação das etiquetas nos sistemas RFID através de simulação computacional. A análise desta influência mostrou os materiais que apresentam maiores interferências na leitura de sistemas RFID. A Figura 4 apresenta o arranjo utilizado na simulação.

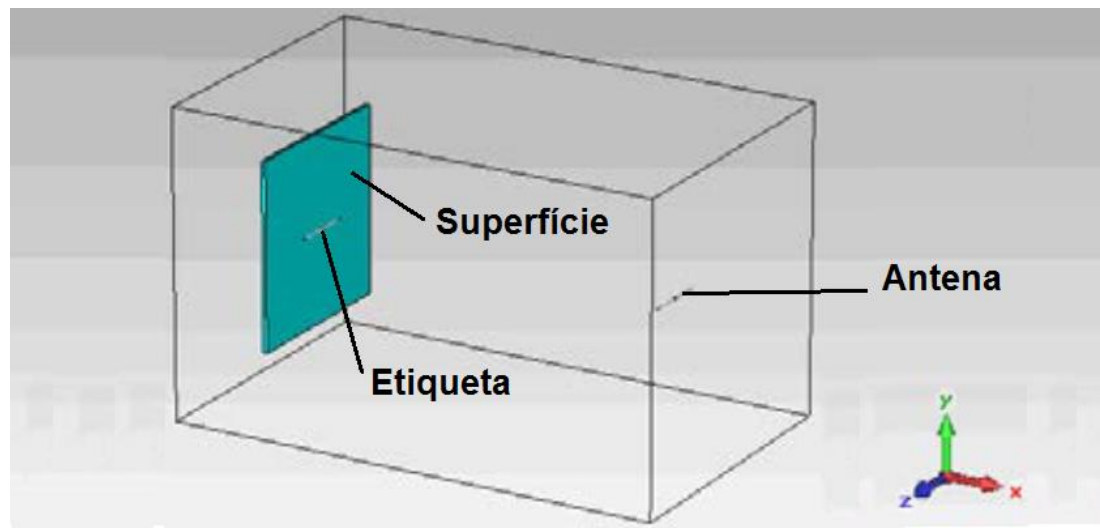

Figura 4. Modelo de Simulação [12].

\section{SISTEMA DE LOCALIZAÇÃO DE ETIQUETAS RFID}

Para a simulação das interferências de ambientes reais na localização de objetos em um Ambiente Laboratorial, desenvolveu-se o Sistema de Localização de Etiquetas RFID (SLER). Este sistema é um RTLS que utiliza RSSI e AoA para o rastreamento e localização. 
O rastreamento é feito em duas dimensões, desta forma são informados suas coordenadas no plano cartesiano indicando a localização de uma mesma etiqueta. Para a comparação dos resultados, o sistema mostra um ponto por técnica.

O SLER é composto por dois subsistemas: Físico e Lógico (Figura 5).

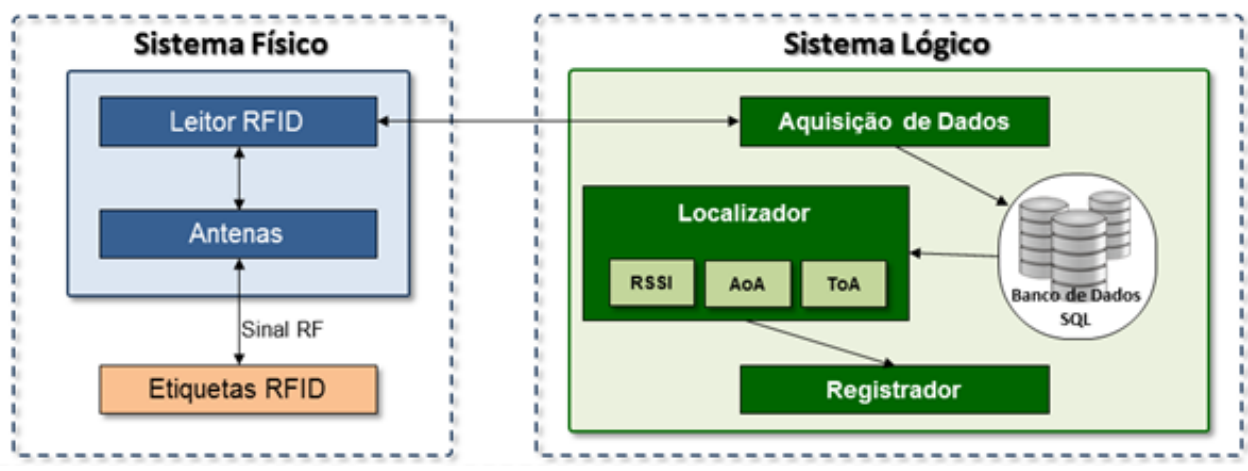

Figura 5. Diagrama de funcionamento do SLER.

\subsection{Subsistema Físico}

O subsistema físico é composto por Leitor, Antenas e Etiquetas RFID (Figura 6) e é responsável por captar as informações do ambiente, através de sinais de radiofrequência e enviá-las para o subsistema Lógico, que tratará as informações. Para que o SLER possa ser reproduzido em aplicações reais empregaram-se equipamentos de mercado.

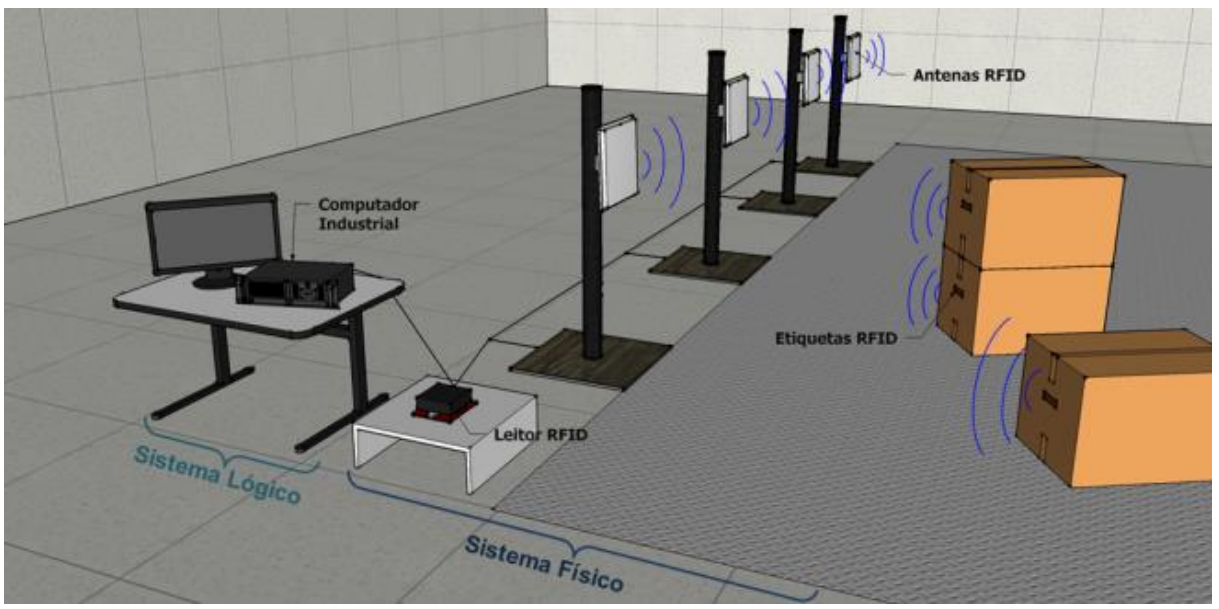

Figura 6. llustração dos equipamentos usados pelo SLER.

Para este trabalho, o leitor utilizado é o Impinj Speedway R420, compatível com o protocolo de interface aérea EPCglobal Gen2 (ISO 18000-63). No leitor são conectadas quatro antenas com $9 \mathrm{dBi}$ de ganho, que operam na faixa de frequência de 902 à $928 \mathrm{MHz}$, mesma faixa de frequência de operação do leitor.

Dois modelos de etiquetas RFID passivas são utilizados, ambos compatíveis com o protocolo de interface aérea EPCglobal Gen2 e operam na mesma faixa de frequência do leitor, conforme descrito na Tabela 1. A etiqueta E02 (Figura 7) possui uma característica diferencial, pois possui a capacidade de operar sobre o metal, Esta 
etiqueta utiliza a superfície metálica em que está instalada como sua antena de transmissão. No entanto, quando não é aplicada sobre metais sua capacidade de resposta à interrogação é reduzida. Neste trabalho, quando é necessário aplicar a etiqueta RFID sobre superfícies metálicas utiliza-se E02, nas demais situações usase E01.

Tabela 1. Modelos de etiquetas RFID e suas características.

\begin{tabular}{cccc}
\hline ID & FABRICANTE & MODELO & $\begin{array}{c}\text { MATERIAL } \\
\text { SUBSTRATO }\end{array}$ \\
\hline E01 & Valid & $\begin{array}{c}\text { VLID105/06 } \\
\text { Chip Monza4E }\end{array}$ & Policarbonato \\
\hline E02 & Xerafy & Data Trak II & $\begin{array}{c}\text { Industry } \\
\text { Grade Polymer }\end{array}$ \\
\hline
\end{tabular}

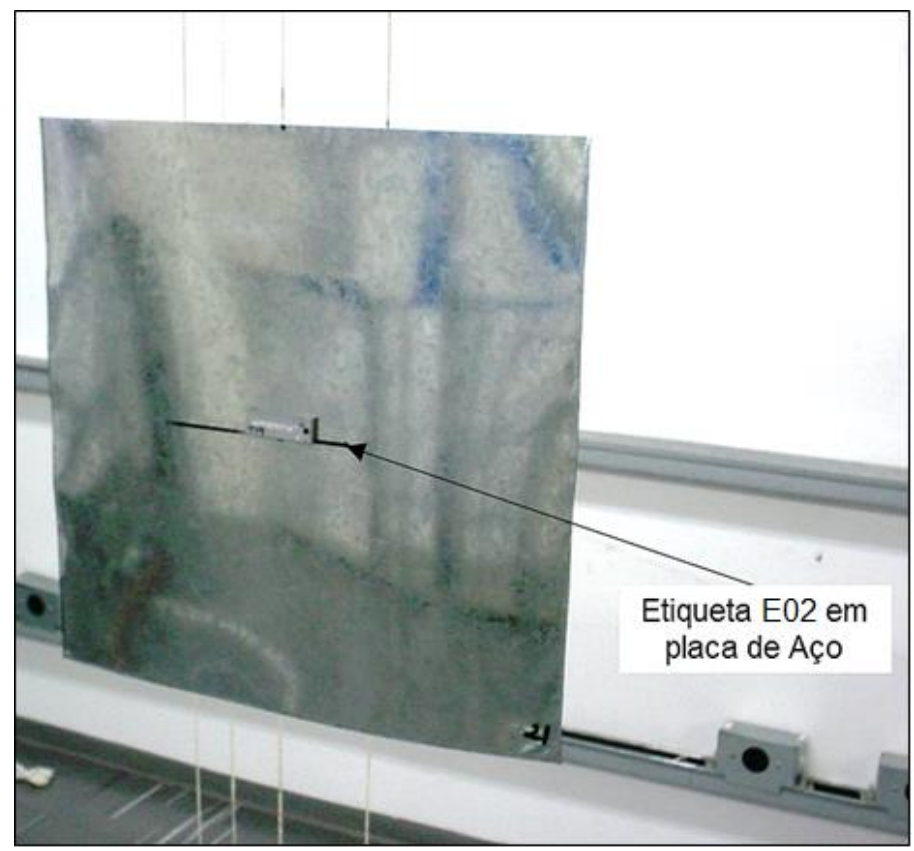

Figura 7. Etiqueta E02 aplicada em superfície metálica.

\subsection{Subsistema Lógico}

O Subsistema Lógico é um software desenvolvido na linguagem C\# que fica hospedado em um computador industrial, o qual se conecta com o leitor RFID via porta Ethernet. Este software é responsável por toda a inteligência do SLER, e executa as seguintes tarefas:

- Aquisição de Dados: Requisita os dados ao leitor RFID. Os sistemas de leitura oferecidos pelos fabricantes de leitor não fornecem todos os dados necessários para o cálculo da localização das etiquetas, apesar destas informações estarem disponíveis para utilização no nível de hardware;

- Localizador: Estima a posição das etiquetas RFID do ambiente no plano cartesiano $(x, y)$ para as duas técnicas previstas no SLER;

- Registrador: Com os dois valores de localização calculados para cada uma das etiquetas, faz a comparação entre as técnicas. 
Durante a tarefa de Localização, o Subsistema Lógico estima a posição das etiquetas para as técnicas RSSI e AoA, utilizando os métodos propostos por Zhang, Li e Amin [11]; e; Zhou e Zhang [6], respectivamente.

\section{TESTES E RESULTADOS}

Nesta seção são apresentados o ambiente laboratorial, métodos de ensaio e resultados experimentais do SLER.

\subsection{Ambiente Laboratorial}

Para verificar as influências ambientais nas técnicas de rastreamento indoor de etiquetas RFID, o SLER será montado para estimar a localização de etiquetas RFID no plano cartesiano, conforme o esquema apresentado na Figura 8.



Figura 8. Distribuição dos equipamentos no Laboratório.

A Figura 9 mostra a presença de 8 anteparos (mostrados como ap) a frente das etiquetas RFID, usados para verificar a influência de obstáculos na comunicação entre etiquetas e antenas RFID, baseando-se no método de Bleda et al. [7]. Também é mostrada a presença de 8 placas (mostrados como pl) sobre as quais a etiqueta RFID é aplicada, baseando-se no método de Mei e Yue [12], para verificar a influência dos materiais usados na fabricação das superfícies dos objetos.

Serão usados anteparos e placas de diferentes materiais, de modo a simular as características do ambiente industrial.

\subsection{Ambiente Industrial}

O ambiente industrial proposto neste trabalho compreende as seguintes indústrias: metalúrgica, automotiva, eletrodomésticos e eletroeletrônicos. Nestes locais, o RFID pode ser usado para o controle do processo produtivo, identificando e localizando item a item. 
Nestas indústrias, os obstáculos na comunicação são gerados basicamente por metais e as etiquetas são fixadas no metal ou em algumas superfícies plásticas [10]. Para simular esta situação no laboratório utilizaram-se anteparos e placas compostos dos materiais da Tabela 2.

Tabela 2. Anteparos e placas para o Ambiente Industrial.

\begin{tabular}{c|c}
\hline ITEM & MATERIAIS \\
\hline Anteparo $(a p)$ & Aço e alumínio. \\
\hline Placa $(p)$ & Aço, alumínio, policarbonato e PVC. \\
\hline
\end{tabular}

\subsection{Métodos de Ensaio}

Como existe uma lista de materiais de placas e anteparos para o Ambiente Industrial, de modo a abranger todas as combinações possíveis entre estes, o ensaio foi composto de 14 configurações, conforme Tabela 3.

Tabela 3. Configurações para o Ensaio.

\begin{tabular}{c|c|c}
\hline CONFIGURAÇÕES & MATERIAL ANTEPARO & MATERIAL $P$ LACA \\
\hline 1 & Ausentes & Aço \\
\hline 2 & Ausentes & Alumínio \\
\hline 3 & Policarbonato & Ausentes \\
\hline 4 & PVC & Ausentes \\
\hline 5 & Aço & Ausentes \\
\hline 6 & Alumínio & Ausentes \\
\hline 7 & Policarbonato & Aço \\
\hline 8 & Policarbonato & Alumínio \\
\hline 9 & PVC & Aço \\
\hline 10 & PVC & Alumínio \\
\hline 11 & Aço & Aço \\
\hline 12 & Aço & Alumínio \\
\hline 13 & Alumínio & Aço \\
\hline 14 & Alumínio & Alumínio \\
\hline & &
\end{tabular}

Segundo Everton [13], o erro de localização (exatidão) é usado como métrica para comparação do desempenho dos sistemas de localização, sendo calculado através da distância Euclidiana $(E c)$ entre a localização estimada e a posição real. Logo, utiliza-se esta métrica para a análise comparativa do estudo proposto.

Para sintetizar o resultado em um único valor numérico, a exatidão da localização é demonstrada por meio da raiz do erro médio quadrático (Root Mean Square Error RMSE), mostrada na Equação 1.

$$
R M S E=\sqrt{\frac{\sum_{t=1}^{k}\left(\widehat{x_{t}}-x_{t}\right)^{2}+\left(\widehat{y_{t}}-y_{t}\right)^{2}}{k}}
$$

Em que $(\hat{x}, \hat{y})$ representam a média das coordenadas estimadas pelo SLER, enquanto que $(x, y)$ as coordenadas reais e $k$ é o número de posições. 


\subsection{Resultados Experimentais}

O gráfico da Figura 9 mostra o resultado do RMSE dos 8 pontos de medição (P11, P12, P13, P14, P21, P22, P23 e P24) para cada uma das configurações apresentada na Tabela 2. A linha em azul com um losango apresenta o resultado utilizando a técnica RSSI e a linha em vermelho com um retângulo apresenta o resultado utilizando a técnica AoA.

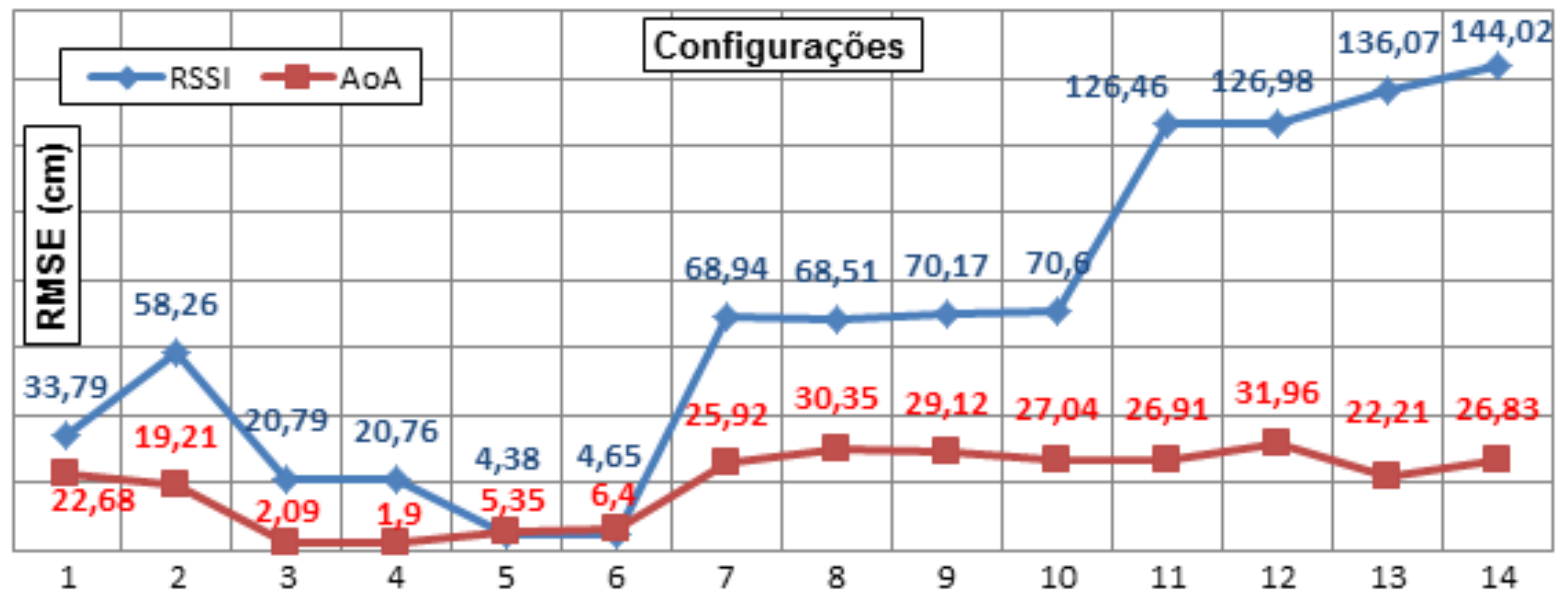

Figura 9. Comparação do RMSE para as configurações.

De maneira geral, a técnica AoA é mais precisa, das 14 configurações, obteve-se o menor erro para 12. Porém, quando consideramos apenas a influência das superfícies de aplicação das etiquetas (placas), ou seja, sem a presença de obstáculos (anteparos), a diferença entre os erros para as duas técnicas é atenuada ficando menor que $2 \mathrm{~cm}$. Como evidência a Figura 10 mostra o RMSE dos pontos P11, P12, P13 e P14 para as configurações 3, 4, 5 e 6 . Nestas configurações, para os primeiros pontos de medição, tem-se somente a presença da superfície de aplicação das etiquetas causando interferência.

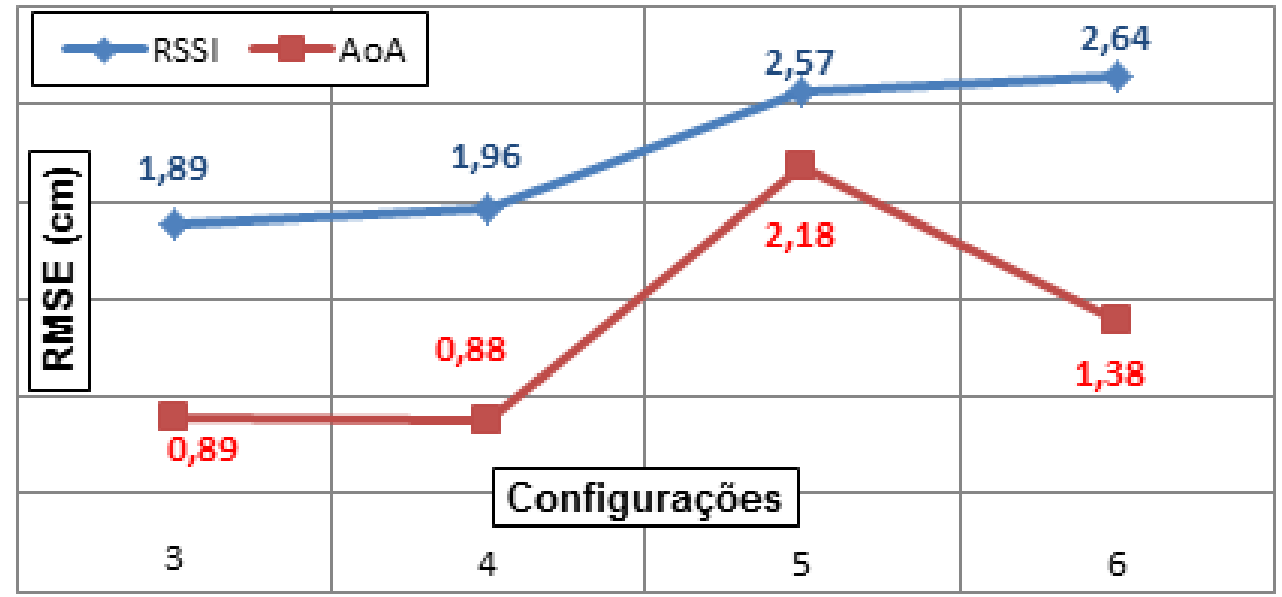

Figura 10. Comparação do RMSE dos pontos $\mathrm{P} 11, \mathrm{P} 12$, $\mathrm{P} 13$ e $\mathrm{P} 14$ para as configurações sem influência de obstáculos. 


\section{CONCLUSÃO}

Os trabalhos realizados por Zhang, Li e Amin [11]; e; Zhou e Zhang [6] apresentaram métodos de localização indoor de etiquetas RFID para uma das técnicas de rastreamento, RSSI e AoA, respectivamente. Ambos não consideram as interferências eletromagnéticas do ambiente.

Bleda et al [7] e; Mei e Yue [12] apresentaram métodos para verificar as interferências do ambiente na comunicação de sistemas RFID. Porém, ambos não abordaram a localização de etiquetas, limitando-se a identificação de objetos.

O presente trabalho realizou um estudo comparativo das técnicas de localização indoor de etiquetas RFID para as técnicas AoA e RSSI, de forma a avaliar os efeitos das interferências do ambiente Industrial baseando-se nos métodos apresentados por Bleda et al [7] e; Mei e Yue [12].

De forma geral, a técnica AoA apresentou a maior exatidão. Portanto, esta é a mais indicada para aplicações no ambiente industrial. Isto ocorre, pois ela é menos influenciada pelos obstáculos entre antenas e etiquetas RFID. No entanto, se considerarmos isoladamente a influência da superfície de aplicação da etiqueta RFID, nota-se que os erros da técnica RSSI ficam no mesmo patamar. Consequentemente, em um cenário com poucos obstáculos na comunicação, pode-se considerar sua utilização.

Os resultados obtidos com este trabalho podem ser complementados em propostas futuras:

- uma análise da localização indoor considerando objetos em movimento. Neste trabalho, se considerou objetos estáticos;

- realização de ensaios em áreas maiores, este trabalho ficou limitado as dimensões do laboratório;

- realização de ensaios com superfícies maiores para aplicação de etiquetas de modo a verificar a relação entre o erro na localização e as dimensões das áreas das superfícies dos objetos;

- o desenvolvimento de um sistema de localização que selecione a melhor técnica (RSSI e AoA) para cada caso de forma automática, através da identificação da presença de obstáculos. De modo a aproveitar os pontos positivos e mitigar os negativos de ambas as técnicas.

\section{REFERÊNCIAS}

1. Flavio Lucas da Rosa, Carlos Fernando Jung, Eduardo José Müller, Patricia Hiromi lida CS ten C. No Geintec - Gestão, Inovação e Tecnol. 2015;5:586-99.

2. Leal AG, Santiago A, Miyake MY, Noda MK, Pereira MJ, Avanco L. Integrated environment for testing loT and RFID technologies applied on intelligent transportation system in Brazilian scenarios. 2014 IEEE Brasil RFID [Internet]. São Paulo: IEEE; 2014. p. 22-4.

3. Hua M-C, Peng G-C, Lai Y-J, Liu H-C. Angle of Arrival Estimation for Passive UHF RFID Tag Backscatter Signal. Green Computing and Communications (GreenCom), 2013 IEEE and Internet of Things (iThings/CPSCom), IEEE 
International Conference on and IEEE Cyber, Physical and Social Computing. Beijing: IEEE; 2013. p. 1865-9.

4. Li Z, Zhou Z, He C, Huang X. Advances in RFID-ILA: The past, present and future of RFID-based indoor location algorithms. Proceedings of the 2012 24th Chinese Control and Decision Conference, CCDC 2012. Taiyuan: IEEE; 2012. p. 3830-5.

5. Brennan D, Kolaja J. Real time location system using passive UHF RFID. Proceedings of the 2014 15th International Carpathian Control Conference (ICCC) [Internet]. Velke Karlovice: IEEE; 2014. p. 58-62.

6. Zhou J, Zhang H, Mo L. Two-dimension localization of passive RFID tags using AOA estimation. 2011 IEEE International Instrumentation and Measurement Technology Conference [Internet]. IEEE; 2011 [cited 2015 May 8]. p. 1-5.

7. Bleda AL, Santa G, Jara AJ, Maestre R, Gomez Skarmeta A. Evaluation of the electromagnetic absorption in furniture for the integration of UHF-RFID tags. 2011 IEEE International Conference on RFID-Technologies and Applications, RFID-TA 2011. Sitges: IEEE; 2011. p. 81-8.

8. Bill Glover HB. RFID Essentials. O'Reilly Media; 2006. 278 p.

9. Avanço L. Um Sistema de Detecção de Intrusão em RFID para ataques do tipo Jamming. 2015.

10. Hessel F, Villar RSG, Dias RR de F, Baladei SDP. Implementando RFID na Cadeia de Negócios. $3^{\mathrm{a}}$ ed. Porto Alegre: ediPUCRS; 2013. 344 p.

11. Zhang Y, Li X, Amin M. Principles and Techniques of RFID Positioning. RFID Syst Res Trends Challenges. 2010;389-415.

12. Mei LH, Yue WJ. Influence of UHF Tags in the Different Material Surface to RFID System. Antennas and Propagation (APCAP), 2014 3rd Asia-Pacific Conference on. Harbin: IEEE; 2014. p. 713-5.

13. Everton LB. Sistema Híbrido de Localização Indoor baseado em RFID e análise visual. Pontifícia Universidade Católica do Rio Grande do Sul (PUC-RS); 2015. 